\title{
SALESPEOPLE QUALITY OF LIFE : INTRODUCING COBE (COMPREHENSIVE BURNOUT AND ENGAGEMENT) MODEL
}

\author{
Sandrine Hollet-Haudebet, IRG University Paris-Est, France
}

\begin{abstract}
Of the various organizational theories developed over the last twenty years, several approaches have been taken up, among them Siegrist's (1996) effort-reward imbalance theory, which defines the sources of job stress in terms of the mismatch between high costs and small gains. The study set out to investigate the relationships between effort/reward, job burnout and work engagement in a salesforce context. The Comprehensive Burnout and Engagement Model (named COBE model) underlying that that burnout develops in response to excessive job demands and diminished job resources (Schaufeli and Bakker, 2004). An empirical study was conducted with 221 salespeople. Results from SEM methods support the COBE model (Schaufeli and Bakker, 2004), burnout is strongly associated to high effort and low reward as well as work engagement. The findings show that there is a significant link in an imbalance situation with the level of engagement, the positive antithesis of professional burnout and burnout. Whether effort is high or the rewards are perceived as low, the absence of reciprocity creates adverse situations for salespeople. Future directions of research are provided.
\end{abstract}

References available upon request 